\title{
Simulación Hidrológica del Caudal del Estero Batuco en la Microcuenca Agroforestal Batuco (Ránquil, Región del Ñuble, Chile) bajo Condiciones Climáticas Presentes y Futuras.
}

\author{
Hormazábal, Víctor ${ }^{1 *}$; Vargas, Víctor 2 ; Abarca-del-Río, Rodrigo1; Little, Christian2; Rivera, Diego3; \\ Carrasco, Noelia ${ }^{4}$ y Villalobos, Enrique ${ }^{2}$ \\ ${ }^{1}$ Departamento de Geofísica, Fac. Ciencias Físicas y Matemáticas, Universidad de Concepción, Chile; vihormazabal@udec.cl \\ 2 Instituto Forestal, Chile. \\ ${ }^{3}$ Facultad de Ingeniería, Universidad del Desarrollo, Chile. \\ ${ }^{4}$ Departamento de Historia, Facultad de Humanidades, Universidad de Concepción, Chile. \\ *Autor para correspondencia.
}

https://doi.org/10.52904/0718-4646.2021.546

Recibido: 29.06.2021; Aceptado 28.07.2021

\section{RESUMEN}

Se estima el comportamiento del caudal del estero Batuco, principal afluente de la microcuenca agroforestal Batuco en la Comuna de Ránquil, entre enero de 1975 y diciembre de 2064, en el escenario del cambio climático RCP8.5. Para tal efecto se simuló hidrológicamente la microcuenca Batuco, usando el modelo y software en versión gratuita WEAP. La información de entrada del modelo para estimar el caudal simulado provino de distintas bases de datos, tanto in situ como de reanálisis cercano a la zona de estudio, así como de datos aproximados del consumo (habitantes y cultivos). Se crearon series temporales para la precipitación y temperatura con los datos in situ, para posteriormente usarse como base para aplicar una corrección de sesgo a los datos de reanálisis Arclim.

Los resultados son una primera aproximación que podrá ser mejorada cuando se pueda calibrar in situ con datos de caudal observado, los cuales actualmente no existen para la zona de estudio. No obstante, evidencian tendencias claras a la reducción sostenida de los caudales.

Se determinó que el caudal simulado del estero Batuco marca una pendiente negativa en el periodo de estudio (19752064) de $\approx-6,3 \mathrm{~L} / \mathrm{s}$ por década, que se manifiesta también para todas las temporadas del año. Las anomalías estandarizadas de los promedios por cada 10 años, muestran en el periodo de 1980 un valor $\approx 1,2$ desviaciones estándar con respecto a la media de la serie, mientras que en el periodo del 2060 se alcanza un valor $\approx-1,5$. Las anomalías estandarizadas de las pendientes por cada 10 años sugieren un comportamiento cíclico, donde su amplitud disminuye con el tiempo para toda la serie y las diferentes temporadas del año.

Palabras clave: Microcuenca agroforestal Batuco, WEAP, RCP8.5.

\section{SUMMARY}

This study estimates the behavior of the flow of the main tributary (Estero Batuco) of the drainage network of the Batuco agroforestry micro-basin in the commune of Ránquil, between January 1975 and December 2064, in the climate change scenario RCP8.5. For this purpose, the Batuco micro-basin was hydrologically simulated, using the free version WEAP model and software. For the model inputs, information was sought from different databases, both in situ and from reanalysis close to the study area. Demand data were also included, such as the approximate consumption of the inhabitants and the silvo-agricultural crops. Time series for precipitation and temperature were created with the in-situ data, to be used later as a basis for applying a bias correction to the Arclim reanalysis data.

The results are a first approximation that can be improved when it can be calibrated in situ with observed flow data, which currently do not exist for the study area. However, they show clear trends towards a sustained reduction in flows.

It was determined that the simulated flow of the Estero Batuco marks a negative slope in the study period $(1975-2064)$ of $\approx$ $-6.3 \mathrm{~L} / \mathrm{s}$ per decade, which also manifests itself for all seasons of the year. The standardized anomalies of the averages for every 10 years show in the 1980 period a value of $\approx 1.2$ standard deviations with respect to the series mean, while in the 2060 period a value of $\approx-1.5$ is reached. The standardized anomalies of the slopes for every 10 years suggest a cyclical behavior, where their amplitude decreases with time for the entire series and the different seasons of the year.

Keywords: Agroforestry microbasin Batuco, WEAP, RCP8.5 


\section{INTRODUCCIÓN}

El recurso hídrico es un bien esencial no solo para el consumo humano, sino también para la industria, la agricultura y la minería, entre otros, pero no solo involucra al hombre o sus actividades productivas sino que define el comportamiento del ecosistema, donde pequeños cambios en la distribución de este recurso pueden causar importantes impactos (IPCC, 2014).

Actualmente existe a escala global una importante redistribución espacio/temporal de las precipitaciones, donde las zonas húmedas se están volviendo más húmedas y las zonas secas, más secas (Trenberth, 2011). El periodo de sequía observable en Chile desde el año 2009 (Garreaud, 2014), posiblemente atribuible a una combinación de forzantes externas (antropogénica entre ellas) y a la variabilidad natural (Boisier et al., 2016), podría estar implicando que la disponibilidad de agua dentro de las cuencas hidrográficas del país también podría estar cambiando (e.g., Bozkurt et al., 2017).

A nivel de subcuenca, una de las zonas productivas de la comuna Ránquil se concentra en la microcuenca de Batuco (Figura 1). En este tipo de zonas productivas predomina la extracción de aguas subterráneas y en menor medida la existencia de vertientes, por lo mismo el sistema agrícola depende principalmente de la capacidad de abastecimiento de los acuíferos, lo que lo hace susceptible a la variación anual e interanual de los mismos (SIMOL et al., 2021).

SIMOL et al. (2021) determinan que la comuna de Ránquil presenta un índice de vulnerabilidad ante la sequía de 0,75 , es decir críticamente vulnerable al cambio climático ${ }^{1}$, valor que sería extrapolable a toda la microcuenca Batuco. El índice de vulnerabilidad proporciona una visión global del territorio, ya que incorpora aspectos ambientales (índices de aridez, presión sobre los recursos hídricos y riesgos de desertificación), aspectos productivos (producción agrícola y ganadera expuesta, diversificación productiva y seguridad de riego tecnificado) y aspectos socioeconómicos (población expuesta, índices de pobreza, acceso al agua potable, desempleo entre otros) (Ministerio de Agricultura et al., 2016).

Vargas et al. (2019) determinaron que, en términos de calidad y cantidad del recurso hídrico, el sector de Batuco depende en gran medida del régimen de las temperaturas y las precipitaciones, así como de las prácticas agrícolas y forestales que se realizan en las zonas aguas arriba (zonas elevadas). En términos de producción, la población local trabaja en agricultura y viticultura a pequeña escala. De las 284 personas que viven en la zona de Batuco, solo dos tienen acceso a agua potable y un tercio de la población no tiene alcantarillado (Municipalidad de Ránquil, 2017).

SIMOL et al. (2021) también determinaron que el abastecimiento actual de agua en la zona de Batuco es a través de un sistema de captación superficial, sin sistema de tratamiento. Se encuentra en proceso (2021) la ejecución de obras de proyecto de agua potable rural (APR).

Fernández et al. (2021) mencionan que un conocimiento insuficiente de la hidrología de una cuenca puede provocar deficiencias en el suministro de agua y no satisfacer las demandas de agua de los usuarios. Es necesario conocer la evolución del balance hídrico, especialmente durante las temporadas secas, y desarrollar estrategias de asignación y manejo. Los mismos autores indican que las evaluaciones a escala de cuenca proporcionan información esencial para la gestión de los recursos hídricos, en particular para predicciones del suministro y la demanda de agua.

Una manera de caracterizar las cuencas y estimar su balance hídrico es a través de modelos o simulaciones. Estos modelos además de intentar representar la realidad hídrica en el presente, también permiten explorar su comportamiento en el pasado y hacer proyecciones en el futuro (e.g., Davie, 2008). Al respecto, se han realizado diversos estudios relacionados con recursos hídricos en el mundo, utilizando modelos hidrológicos (e.g., Maliehe y Mulungu, 2017; Condom et al., 2011; Bhave et al., 2014; Duque y Vázquez, 2015), y algunos también en Chile (e.g., Fernández-Alberti et al., 2021; Mena, 2009; Río La Mura, 2015; Vargas et al., 2012).

Por esta razón es interesante evaluar y caracterizar las cuencas hidrográficas para conocer su potencial hídrico, analizando las distintas variables climatológicas, geográficas, usos de suelo, entre otras, a pequeña y gran escala, y así poder crear escenarios para su correcta gestión (e.g., Meza et al., 2014).

${ }^{1}$ Los valores de este índice varían entre 0 y 1 , donde 0 no es vulnerable y 1 es muy vulnerable. 
En tal sentido, el objetivo de este trabajo es estimar el caudal del estero Batuco, entre enero de 1975 y diciembre de 2064, explorar el comportamiento de la variabilidad de su promedio cada 10 años y sus pendientes cada 10 años, y profundizar en las temporadas del año. Todo esto se desarrolla en el escenario del cambio climático RCP8.5, el cual es el "peor escenario posible del cambio climático" (IPCC, 2019).

\section{MATERIAL Y MÉTODO}

\section{La Microcuenca de Estudio}

El área de estudio corresponde a la microcuenca Batuco, ubicada en la comuna de Ránquil, en un área de secano interior (territorio sin riego ubicado en el lado oriental de la Cordillera de la Costa de Chile) conocido como Valle del Itata, provincia de Itata, en la región de Ñuble, en el centro-sur de Chile. Se ubica aproximadamente en las coordenadas latitud -36,583 y longitud -72,648.

La microcuenca de Batuco (Figura 1) posee un área de aproximadamente $5,37 \mathrm{~km}^{2}$ y se ubica principalmente en la Sub-cuenca Ñuble Bajo y Estero Chudal, la cual a su vez pertenece a la Cuenca del Río Itata. En la zona alta de la microcuenca ubicada al sur existen plantaciones agroforestales que pertenecen a pequeños propietarios forestales. En la parte media y baja se encuentran las plantaciones forestales (Pinus radiata D. Don. y Eucalyptus globulus Labill) que pertenecen a los propietarios de bosques de mediana escala y la gran empresa forestal (Vargas et al., 2019).

El caudal principal de la microcuenca, denominado en este estudio Estero Batuco, va de sur a norte hasta la zona de toma de agua, que es donde se ubican las bombas de agua que abastecen a la comunidad de Batuco (Vargas et al., 2019).

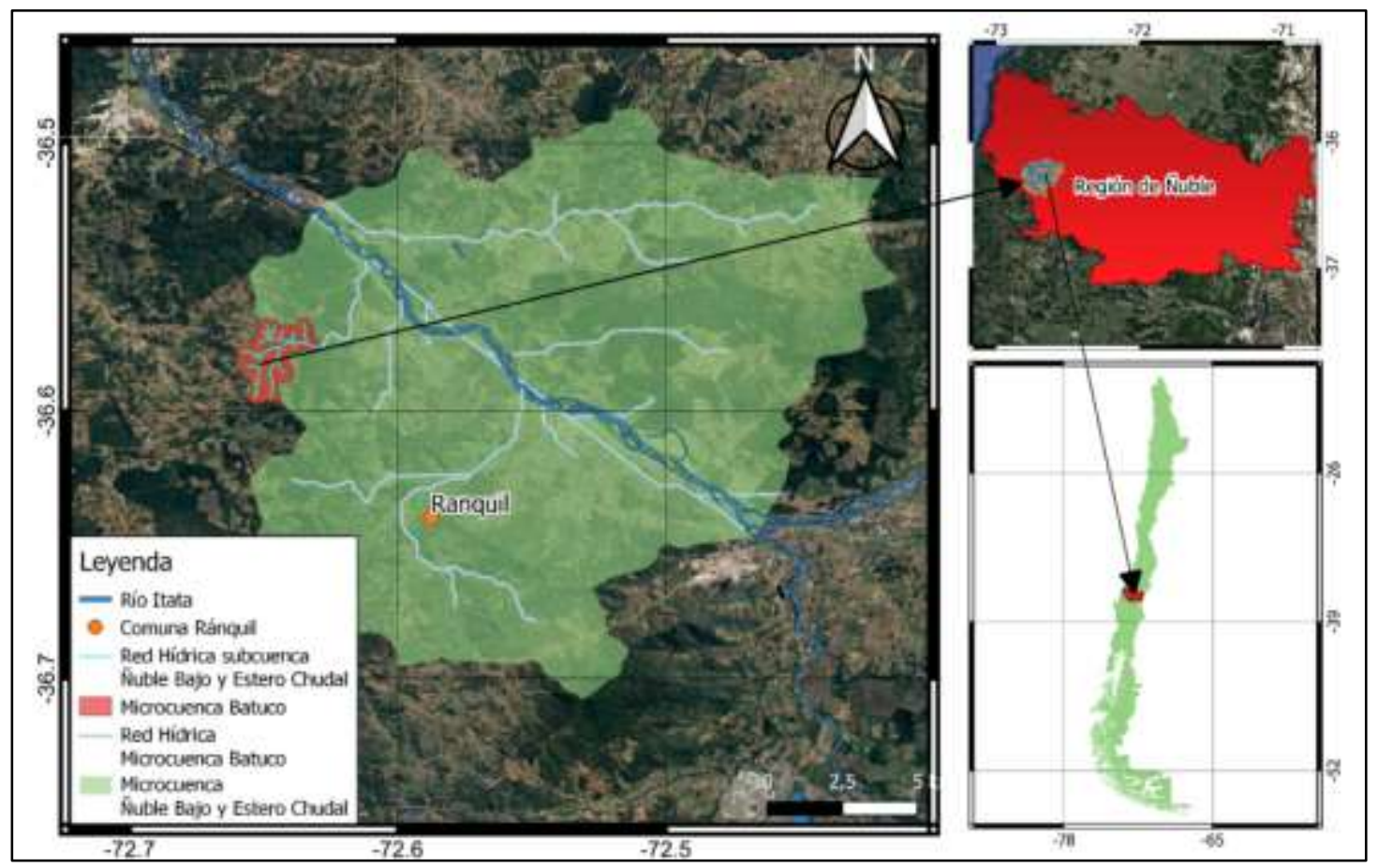

Figura 1. Zona de Estudio.

\section{Información Disponible}

En los alrededores de la zona de estudio, se recolectó información de 23 estaciones de monitoreo hidrometeorológicas. Esta información fue extraída en los sitios web del explorador climático (Center for Climate and Resilience Research (CR)2 et al., 1940-2021) y la red agrometeorológica INIA (Instituto de 
Investigaciones Agropecuarias et al., 2009-2021). Además, se incluyeron 3 estaciones offline a cargo del Instituto Forestal, las cuales se instalaron en la microcuenca Batuco. Esta información se resume en el Cuadro 1.

Cuadro 1. Lista de Estaciones Meteorológicas Usadas.

\begin{tabular}{|c|c|c|c|c|c|c|c|c|}
\hline $\mathbf{N}$ & Nombre & Latitud & Longitud & Altitud & $\begin{array}{c}\text { Fin } \\
\text { observación }\end{array}$ & $\begin{array}{c}\text { Inicio } \\
\text { observación }\end{array}$ & $\begin{array}{l}\text { Variables } \\
\text { extraídas }\end{array}$ & Fuente \\
\hline 1 & Millauquén & $-36,3183$ & $-72,0400$ & 152 & 31-03-2017 & 01-02-1992 & Ppt & DGA \\
\hline 2 & $\begin{array}{l}\text { San Agustín de } \\
\text { Puñual }\end{array}$ & $-36,4213$ & $-72,3944$ & 138 & 31-03-2017 & 01-01-1993 & Ppt & DGA \\
\hline 3 & Coelemu & $-36,4750$ & $-72,6882$ & 40 & $31-03-2017$ & $01-10-1961$ & Ppt & DGA \\
\hline 4 & Dichato & $-36,5456$ & $-72,9311$ & 11 & 31-03-2017 & 01-06-1980 & Ppt & $\overline{D G A}$ \\
\hline 5 & San Fabián & $-36,5835$ & $-71,5246$ & 465 & 31-03-2017 & 01-10-1956 & Ppt & DGA \\
\hline 6 & Chillán Viejo & $-36,6319$ & $-72,1267$ & 115 & $29-11-2017$ & $01-01-1977$ & Ppt & DGA \\
\hline 7 & Rafael & $-36,6364$ & $-72,8489$ & 294 & 31-03-2017 & 01-01-1993 & Ppt & $\overline{D G A}$ \\
\hline 8 & $\begin{array}{l}\text { Coihueco } \\
\text { Embalse }\end{array}$ & $-36,6407$ & $-71,7989$ & 314 & 28-02-2017 & 01-01-1900 & Ppt & DGA \\
\hline 9 & Caracol & $-36,6511$ & $-71,3949$ & 610 & $31-03-2017$ & $01-01-1900$ & Ppt & DGA \\
\hline 10 & Nueva Aldea & $-36,6544$ & $-72,4561$ & 32 & $31-03-2017$ & $01-09-1956$ & Ppt, temp & DGA \\
\hline 11 & Camán & $-36,6730$ & $-71,2989$ & 670 & 31-03-2017 & 01-09-1992 & Ppt & $\overline{D G A}$ \\
\hline 12 & Chillancito & $-36,7622$ & $-72,4239$ & 56 & $31-03-2017$ & 01-07-1954 & Ppt & DGA \\
\hline 13 & Mayulermo & $-36,8188$ & $-71,8944$ & 371 & 31-03-2017 & 01-02-1992 & Ppt & $\overline{D G A}$ \\
\hline 14 & Diguillín & $-36,8686$ & $-71,6424$ & 670 & $31-03-2017$ & $01-01-1900$ & Ppt & DGA \\
\hline 15 & Las Trancas & $-36,9108$ & $-71,4792$ & 1242 & 31-03-2017 & $01-12-1961$ & Ppt & DGA \\
\hline 16 & Fundo Atacalco & $-36,9174$ & $-71,5814$ & 740 & 31-03-2017 & $01-10-1930$ & Ppt & $\overline{D G A}$ \\
\hline 17 & Pemuco & $-36,9763$ & $-72,1008$ & 200 & $31-03-2017$ & $31-08-1971$ & Ppt & DGA \\
\hline 18 & Las Cruces & $-37,1114$ & $-71,7650$ & 467 & 31-03-2017 & 01-01-1993 & Ppt & DGA \\
\hline 19 & Cholguán & $-37,1527$ & $-72,0669$ & 230 & 31-03-2017 & 01-09-1956 & Ppt & DGA \\
\hline 20 & Chillán Ad & $-36,5872$ & $-72,0400$ & 151 & $29-11-2017$ & 01-01-1950 & Ppt, temp & DMC \\
\hline 21 & $\begin{array}{l}\text { Carriel Sur } \\
\text { Concepción }\end{array}$ & $-36,7792$ & $-73,7792$ & 12 & $29-11-2017$ & 01-01-1950 & Ppt, temp & DMC \\
\hline 22 & Ninhue & $-36,39$ & $-72,39$ & 91 & $30-04-2021$ & 01-04-2010 & temp & INIA \\
\hline 23 & Portezuelo & $-36,53$ & $-72,36$ & 109 & $30-04-2021$ & $01-04-2010$ & temp & INIA \\
\hline 24 & $\begin{array}{l}\text { Escuela } \\
\text { Checura }\end{array}$ & $-36,5906$ & $-72,6492$ & 308 & $31-01-2021$ & $18-01-2020$ & $\begin{array}{l}\text { Ppt, temp, } \\
\text { V, HR }\end{array}$ & $\begin{array}{l}\text { INFOR/ } \\
\text { UDEC }\end{array}$ \\
\hline 25 & Sra. Silvia & $-36,5740$ & $-72,6063$ & 37 & $31-01-2021$ & $18-01-2020$ & $\begin{array}{l}\text { Ppt, temp, } \\
\text { V, HR }\end{array}$ & $\begin{array}{l}\text { INFOR/ } \\
\text { UDEC }\end{array}$ \\
\hline 26 & Vertiente Abajo & $-36,5753$ & $-72,6291$ & 120 & $31-01-2021$ & $18-01-2020$ & HS & $\begin{array}{l}\text { INFOR/ } \\
\text { UDEC }\end{array}$ \\
\hline
\end{tabular}

(Ppt= precipitación; temp= temperatura; $\mathrm{V}=$ velocidad del viento; $\mathrm{HR}=$ humedad relativa; $\mathrm{HS}=$ humedad del suelo).

También se utilizaron datos de reanálisis hidroclimáticos, los cuales se extrajeron de los sitios web Atlas de Riesgo Climático (Center for Cimate and Resilience Research (CR)2 et al., 1970-2069) (esta base de datos se denominará Arclim de ahora en adelante) y del producto climatológico ERA5 de Climate Copernicus (Copernicus et al., 1979-2021) del tamaño de un pixel, lo cual era suficiente para cubrir el área de la microcuenca en su totalidad.

En el caso de los datos Arclim, estos poseen distintas simulaciones que abarcan desde el año 1970 hasta el año 2069 en el evento del cambio climático RCP8.5, para un periodo de referencia (enero de 1980 hasta diciembre del 2009) y un periodo futuro (enero de 2035 hasta diciembre del 2064) (Center for Climate and Resilience Research (CR)2 et al., 2020).

Estos modelos tienden a promediarse para tener una serie más robusta (e.g., Murphy, 1998). Esta información se resume en el Cuadro 2. 
Cuadro 2. Lista de los Datos de Reanálisis Utilizados.

\begin{tabular}{|c|c|c|c|c|c|c|c|}
\hline \multirow{2}{*}{$\mathbf{N}$} & \multirow{2}{*}{ Fuente } & \multirow{2}{*}{$\begin{array}{c}\text { Tamaño } \\
\text { Pixel }\end{array}$} & \multirow{2}{*}{ Latitud } & \multirow{2}{*}{ Longitud } & \multicolumn{2}{|c|}{ Re análisis / Simulación } & \multirow{2}{*}{ Variables } \\
\hline & & & & & Fin & Inicio & \\
\hline 1 & ARCLIM & $\approx 5 \mathrm{~km} \times 5 \mathrm{~km}$ & $-36,58$ & $-72,62$ & $31-12-2069$ & $01-01-1970$ & Ppt, temp \\
\hline 2 & ERA5 & $\approx 9 \mathrm{~km} \times 9 \mathrm{~km}$ & $-36,50$ & $-72,50$ & $31-12-2015$ & $01-01-2013$ & Tcc \\
\hline
\end{tabular}

Para crear la red hídrica de la microcuenca Batuco se dispuso de un Modelo Digital de Elevación (DEM) del satélite ALOS, donde los productos DEM, ALOS, y PALSAR ofrecidos por Alaska Satellite Facility tienen una resolución espacial de 12,5 x 12,5 m a nivel global (Geophysical Institute of the University of Alaska Fairbanks, 2006-2011).

Para hacer la clasificación de uso de tipo de suelo, se recurrió a un conjunto de imágenes satelitales a diferentes bandas del espectro electromagnético del Sentinel-2, con una resolución espacial de $10 \times 10$ m, correspondientes al 13 de enero del año 2021 a las 14:00 UTC aproximadamente (Copernicus \& European Space Agency ESA, 2015).

También se contó con mediciones de la capacidad de almacenamiento del agua del suelo, aproximadamente en la latitud $-36,5755^{\circ}$ longitud $-72,6295^{\circ}$, entre los $25-60 \mathrm{~cm}$ y $60-110 \mathrm{~cm}$ de profundidad. Estas mediciones corresponden aproximadamente a $725 \mathrm{~m}^{3} / \mathrm{ha}$ y $935 \mathrm{~m}^{3} / \mathrm{ha}$, respectivamente (mediciones tomadas por INFOR).

En la microcuenca Batuco habitan cerca de 650 personas (Municipalidad de Ránquil, 2021). Según se informa en Marticorena, (2020), el consumo diario por persona en Chile es de 172 litros por día. En un ejemplo del SEI (2015), el consumo anual agrícola por hectárea fue de $3.500 \mathrm{~m}^{3}$.

Adicionalmente se contó con una medición del caudal del estero Batuco, obtenida el 24 de diciembre de 2019 por la empresa Forestal Arauco. Esta medición corresponde a 9,86 L/s, dato que no fue utilizado en el análisis y se considera solo como referencia.

\section{Estadísticos y Tratamiento de Datos}

\section{- Anomalías Estandarizadas}

Se usó la anomalía estandarizada z para comparar variables con magnitudes muy dispares (ecuación 1), donde cada variable es adimensional y tiene una media de 0 y una desviación estándar (std) de 1. Se calcula simplemente restando la media muestral de los datos brutos $\mathrm{X}$ y dividiendo por la desviación estándar muestral correspondiente (sx).

$$
z=\frac{X-\bar{X}}{s_{X}}
$$

Una forma de analizar la anomalía estandarizada es como medida de distancia, en unidades de desviación estándar (std), entre un valor de datos y su media. Además, al estandarizar los datos, estos serán adimensionales, lo cual permitiría compararlos con otros datos estandarizados, aunque su origen haya sido diferente (Wilks, 2005).

Estas anomalías estandarizadas se calcularon después de haber calculado promedios cada 10 años y pendientes cada 10 años, sucesivamente (algo similar a una media móvil), abarcando por ejemplo desde enero 1975 hasta diciembre de 1984 para representar el año 1980 y así sucesivamente para las otras décadas (Ver ejemplos en Figura $N^{\circ} 8$ y № 9).

\section{- Pendiente}

Se usó un ajuste de regresión lineal, para estimar la pendiente $p$ entre dos variables (caudal simulado y tiempo en este caso) (ecuación 2): 


$$
p=\frac{n \sum X Y-\sum X Y}{n \sum X^{2}-\left(\sum X\right)^{2}}
$$

Donde:

$$
\begin{aligned}
& \mathrm{p}=\text { pendiente } \\
& \mathrm{n}=\text { número de datos }
\end{aligned}
$$

En general la pendiente en este caso se usará para estimar de manera aproximada la tasa de cambio entre dos variables (es aproximada, porque se omitirá el valor de intersección en la fórmula de regresión lineal).

\section{- Coeficiente de Determinación}

Se usó el Coeficiente de Determinación $\mathrm{R}^{2}$ (ecuación 3) para poder estimar la varianza compartida que existe entre 2 variables $\mathrm{X}$ e $\mathrm{Y}$. Se corresponde con el cuadrado del coeficiente de correlación de Pearson.

$$
R^{2}=\frac{\sigma_{X Y}^{2}}{\sigma_{X}^{2} \sigma_{Y}^{2}}
$$

Donde:

$$
\begin{aligned}
\sigma_{X Y}^{2} & =\text { covarianza de } \mathrm{X} \text { e } \mathrm{Y} \\
\sigma_{X}^{2} & =\text { varianza de } \mathrm{X} \\
\sigma_{Y}^{2} & =\text { varianza de } \mathrm{Y} .
\end{aligned}
$$

\section{Modelo Weap}

Se empleó la versión gratuita del código Water Evaluation And Planning System o WEAP (SEI, 2015), el cual presenta un sistema integrado de planificación de los recursos hídricos, conectando los procesos hidrológicos y sus operaciones en una única plataforma analítica.

\section{- Unidad Hidrológica}

Para efectos de este estudio y teniendo como referencia al Centro de Cambio Global-Universidad Católica de Chile y e Stockholm Environment Institute (2009), se determinó por simplicidad que solo existirá una unidad hidrológica ${ }^{2}$ o catchment, el cual cubriría toda la microcuenca Batuco, ya que la red hídrica converge casi en su totalidad al final de la microcuenca Batuco (de sur a norte), como se muestra en la Figura 1.

\section{- Simulación Hidrológica}

Se utilizó el método de humedad del suelo, que representa el proceso precipitación-escorrentía en una unidad de respuesta hidrológica (en este caso la microcuenca Batuco), por medio de dos estanques, con los cuales se busca reproducir los flujos superficiales y subterráneos (SEI, 2015) a una escala mensual.

Este método unidimensional se basa en funciones empíricas que describen la evapotranspiración, la escorrentía superficial, el escurrimiento subsuperficial (flujo intermedio) y la percolación profunda.

En la Figura 2 se puede apreciar la conceptualización del método y las diferentes ecuaciones y criterios considerados al momento de representar la hidrología en una columna unidimensional.

\footnotetext{
${ }^{2}$ La Unidad hidrológica es un área que separa la cuenca o subcuenca por bandas de elevación
} 


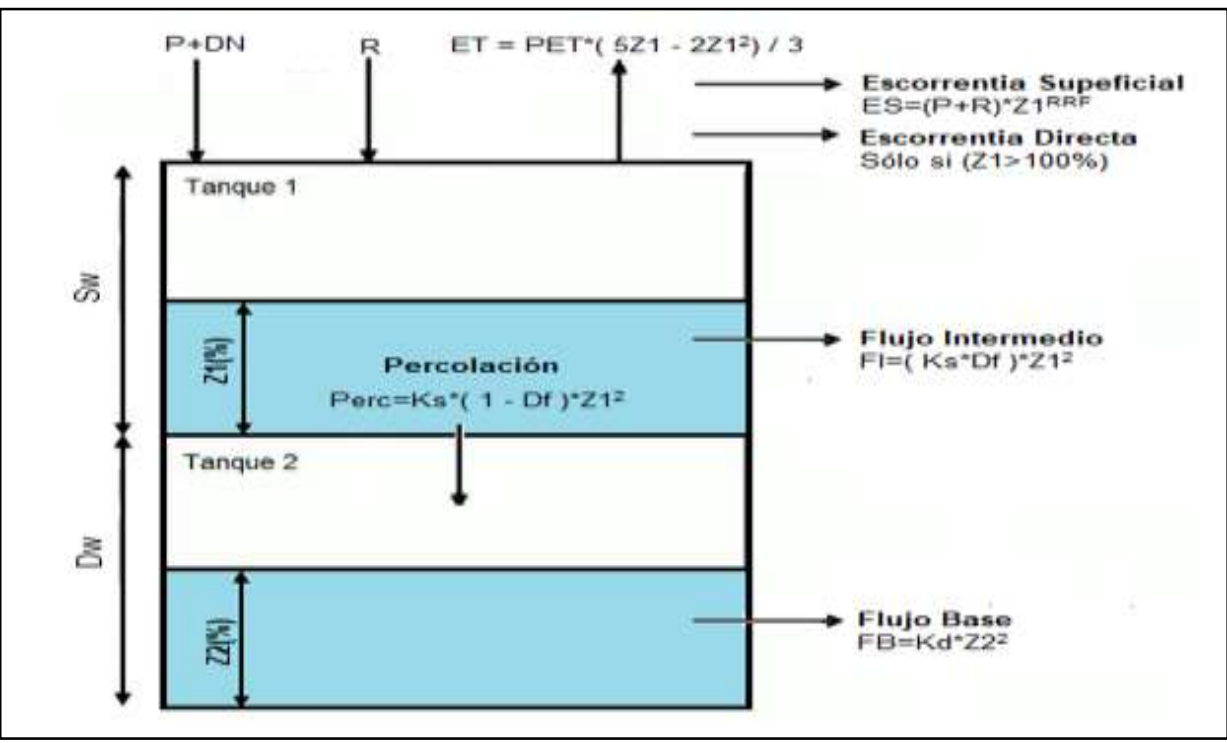

(En base a SEI, 2015)

P precipitación $(\mathrm{mm})$; DN derretimiento de nieve $(\mathrm{mm}) ; \mathbf{R}$ recarga de riego $(\mathrm{mm})$; ET evapotranspiración real (mm); PET evapotranspiración potencial; Z1 porcentaje de agua en el estanque superior (\%); Z2 porcentaje de agua en el estanque inferior (\%); Ks conductividad hidráulica del estanque superior $\left(\mathrm{mm} \mathrm{mes}^{-1}\right)$; Kd conductividad hidráulica del estanque inferior $(\mathrm{mm}$ mes $^{-1}$ ); Df dirección preferencial del flujo (adimensional), que separa el flujo superficial del subterráneo; RRF coeficiente de resistencia a la escorrentía de la cobertura del suelo (adimensional); Sw capacidad de estanque superior $(\mathrm{mm})$; y $\mathbf{D w}$ capacidad del estanque inferior $(\mathrm{mm})$.

Figura 2. Representación Esquemática del Módulo Hidrológico para el Método de Humedad del Suelo en WEAP.

Las ecuaciones (4) y (5) representan el balance en cada estanque (SEI, 2015):

$$
\begin{gathered}
S w \frac{d Z 1}{d t}=\left[P e(t)-P E T(t) \frac{5 Z 1-2 Z 1^{2}}{3}\right]-P e(t) Z 1^{R R F}-D f(K s) Z 1^{2}-K_{2}(1-D f) Z 1^{2} \\
D w \frac{D Z 2}{d t}=K d(1-D f) Z 1^{2}-K d Z 2^{2}
\end{gathered}
$$

Donde:

$$
\begin{aligned}
& \mathrm{P}_{\mathrm{e}}=\text { precipitación más el derretimiento de nieve }(\mathrm{mm}) \\
& \text { Las demás expresiones son las detalladas en la Figura } 2
\end{aligned}
$$

Estas ecuaciones son empíricas y describen la dinámica de flujo que acontece en los estanques, los mismos que conceptualizan los componentes superficiales, subsuperficial y de percolación profunda del ciclo hidrológico.

La evapotranspiración potencial (ecuación 6), se puede descomponer en:

$$
P E T=K c \times E T_{0}
$$

Donde:

$$
\begin{aligned}
& \mathrm{Kc}=\text { coeficiente de cultivo } \\
& E T_{0}=\text { evapotranspiración de cultivo de referencia. }
\end{aligned}
$$


La evapotranspiración potencial del cultivo de referencia ( $\left.E T_{0}\right)$ en mm/día (ecuación 7), se obtiene de la fórmula de Penman-Monteith que es la base del método 56 de la FAO (Allen et al., 1998):

$$
E T_{0}=\frac{(0.408) \delta\left(R_{n}-G\right)+\gamma \frac{900}{T+273} u_{2}\left(e_{s}-e_{a}\right)}{\delta+\gamma\left(1+0.34 u_{2}\right)}
$$

Donde:

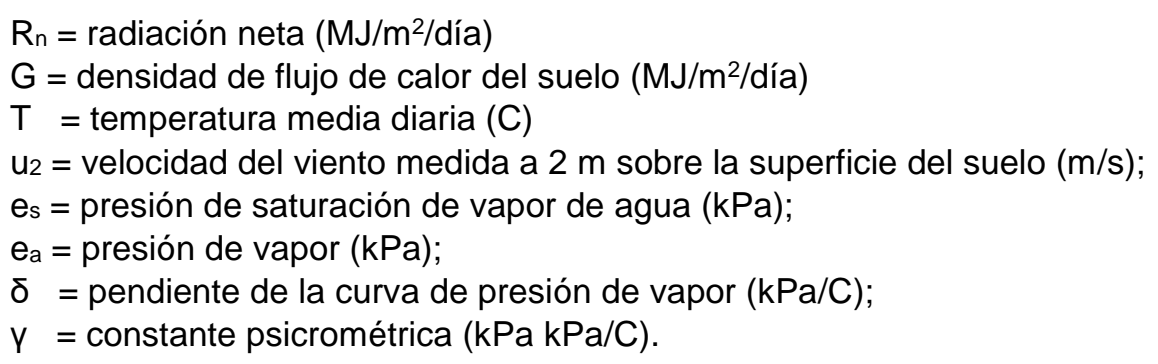

\section{- Demandas Dentro del Modelo}

Una vez obtenido un caudal simulado por el método de la humedad del suelo en un mes determinado, se procedió a utilizar la característica de planificación denominada zonas de demanda, disponible dentro del código WEAP (SEI, 2015), con la intención de obtener una mejor estimación de la variación del caudal total liberado por la microcuenca Batuco en la desembocadura del estero Batuco.

Se agregaron 2 zonas de demanda llamadas zona agrícola y zona urbana. Las características de estas zonas de demanda se detallaron anteriormente en la subsección información disponible de esta misma sección (metodología). Finalmente, el balance hídrico (ecuación 8) queda:

$$
Q_{\text {Simulado }}(t)-Q_{\text {Agrícola }}(t)-Q_{\text {Urbano }}(t)=Q_{\text {total }}(t)
$$

Donde:

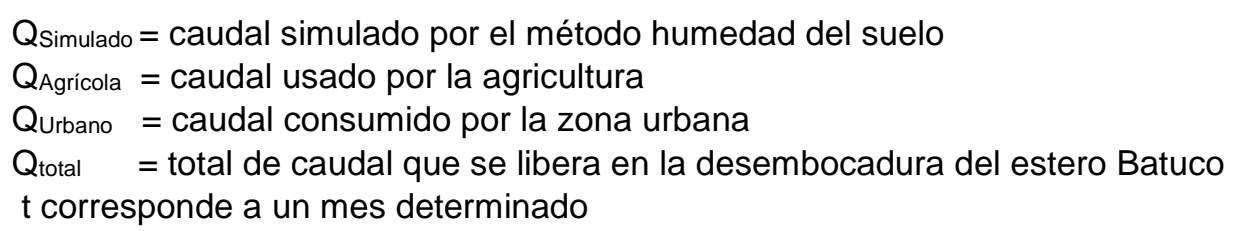

Se debe comentar que en $Q_{\text {simulado }}(t)$ también se incluye el aporte del mes anterior $(t-1)$ que puede producir la microcuenca sin aportaciones de precipitación (flujo base), excepto para el principio de la simulación ( $\mathrm{t}=1)$.

\section{Caracterización Meteorológica y Climatológica}

\section{- Precipitación y Temperatura}

Se utilizó la media de las simulaciones para el evento del cambio climático RCP8.5 disponibles en la plataforma Arclim, desde enero del 1970 hasta diciembre de 2069 con una distribución temporal a escala mensual, para el pixel que cubre la zona de estudio como serie de entrada al modelo WEAP, tanto para la precipitación como para la temperatura (precipitación y temperatura Arclim de ahora en adelante), pero antes de eso se aplicó una corrección de sesgo con los datos in situ de las estaciones de monitoreo hidrometeorológicas, para obtener series más representativas de la zona de estudio.

Para la representatividad de la precipitación y la temperatura en la zona de estudio con los datos in situ, se utilizaron curvas de isoyetas e isotermas, respectivamente, siguiendo la metodología explicada en (Duque y Vázquez, 2015), con una distribución temporal mensual. 
Para la precipitación, los criterios para seleccionar las estaciones (estaciones 1-21 del Cuadro 1) fueron:

i) Que se contara con disponibilidad de datos desde enero del 1996 hasta diciembre del 2015

ii) ii) Que existieran a lo menos 14 años donde se pudiera representar el total de precipitación anual. Luego a partir de la ecuación (9) se calculó la precipitación anual:

$$
P_{(a, e)}=\sum_{m=1}^{12} P_{(m, a, e)}(m)
$$

Donde:

$$
\begin{aligned}
& \mathrm{P}_{(\mathrm{a}, \mathrm{e})}=\text { precipitaciones acumuladas en un año a en una estación e } \\
& \mathrm{P}_{(\mathrm{m}, \mathrm{a}, \mathrm{e})}=\text { precipitación mensual en un mes } \mathrm{m} \text {, en un año a y una estación e }
\end{aligned}
$$

Una vez ya seleccionadas las estaciones hidrometeorológicas a usar en base a la ecuación (9), se calculó la media temporal de los 14 o más datos de promedios anuales.

Luego a partir de la ecuación (10) se calculó la media de las precipitaciones anuales de cada estación seleccionada:

$$
P_{(p a, e)}=\frac{\sum_{a=1}^{n} P_{(a, e)}(a)}{n}
$$

Donde:

$$
\begin{aligned}
& P_{(p a, e)}=\text { media de las precipitaciones acumuladas anuales en una estación } \\
& P_{(a, e)}=\text { precipitación anual en el año a y estación e } n \text { es el número de años }(n \geq 14) \\
& n \quad=\text { número de años }(n \geq 14)
\end{aligned}
$$

Después de calcular la media de las medias anuales en cada estación meteorológica, se usó un sistema de información geográfica (SIG) ${ }^{3}$ para generar un mapa con la interpolación de la media de las precipitaciones acumuladas anuales y las isoyetas cada $50 \mathrm{~mm}$, en la región del Nuble y la parte norte de la región del Bio Bio (Figura 3).

Siguiendo la metodología usada en (Duque y Vázquez, 2015), en la parte superior derecha de la Figura 3 , se observa que transita una isoyeta anual de $1000 \mathrm{~mm}$, por lo que se considerará este valor como representativo de la zona de estudio.

Se escogió la estación pluviométrica Coelemu (símbolo amarillo en Figura № 3 o estación número 3 del Cuadro $\mathrm{N}^{\circ}$ 1) como la estación de referencia la cual marca una precipitación anual de $843.6 \mathrm{~mm}$.

\footnotetext{
${ }^{3}$ Qgis
} 


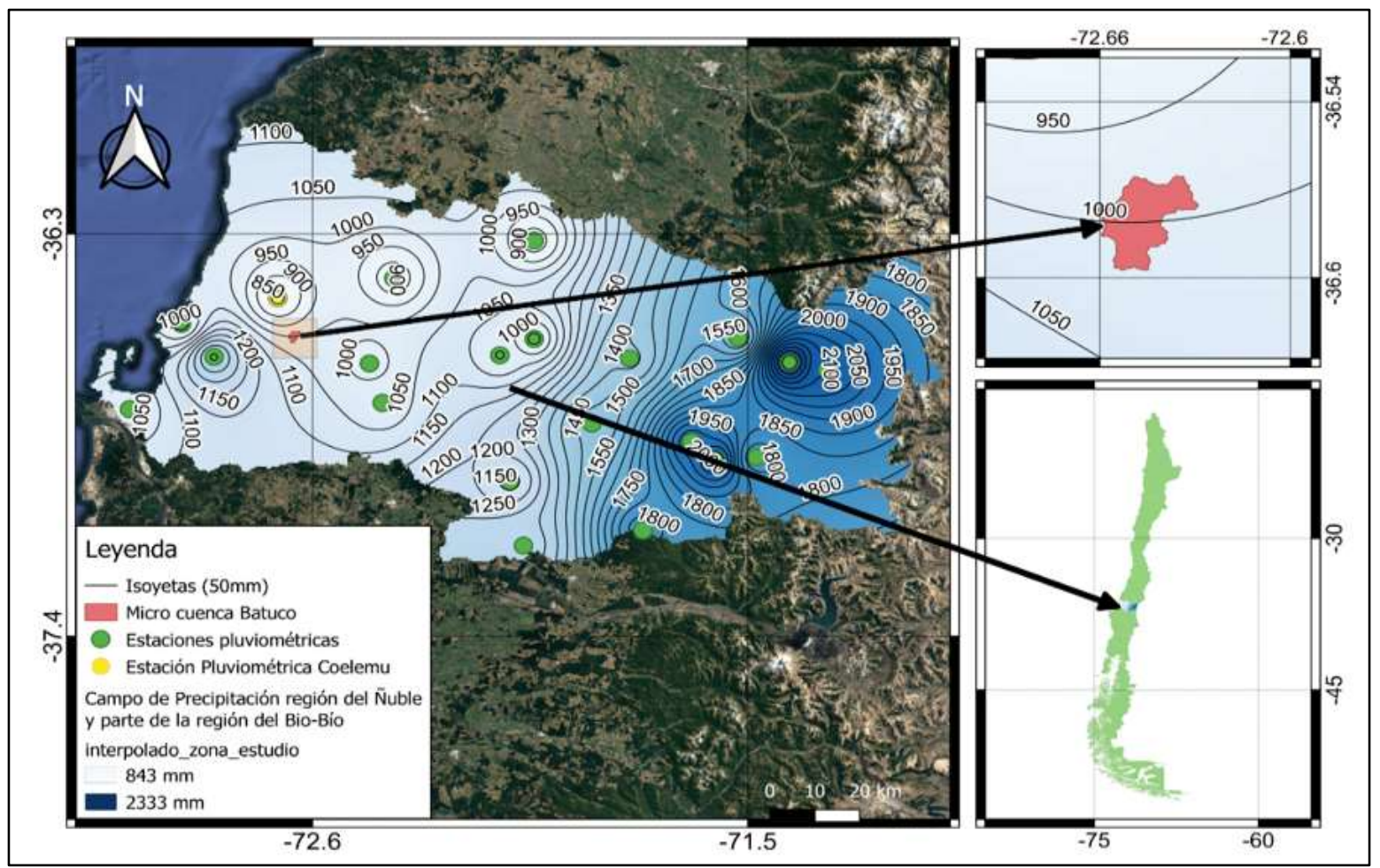

Figura 3. Interpolación de la Precipitación Anual por el Método de Isoyetas) en la Región de Nuble y la Parte Norte de la Región del Biobío.

A partir de la ecuación (11) se calculó la precipitación a escala mensual en Batuco:

$$
P b_{(m, a)}=P c_{(m, a)} x \frac{P b_{(p a)}}{P c_{(p a)}}
$$

Donde:

$\mathrm{Pb}_{(\mathrm{m}, \mathrm{a})}=$ precipitación en Batuco (zona de estudio) en el mes $\mathrm{m}$ y el año $\mathrm{a}$;

$\mathrm{Pc}_{(\mathrm{m}, \mathrm{a})}=$ precipitación en el mes $\mathrm{m}$ y el año $\mathrm{a}$, de la estación de referencia (Coelemu);

$\mathrm{Pb}_{(\mathrm{pa})}=$ precipitación anual representativa (isoyeta en batuco de la Figura 3);

$\mathrm{PC}_{(\mathrm{pa})}$ es la precipitación anual de la estación de referencia (Coelemu).

Para aumentar la confianza en la serie de precipitación creada a partir del método de isoyetas, se comparó con una reconstrucción reciente de series de precipitaciones desarrolladas por Alvial et al. (2020), la cual creó una base de datos de precipitación en alta resolución $(1 \mathrm{~km})$ mediante la utilización de una reducción de escala dinámico-geoestadístico.

Para construir esta base de datos se utilizó tanto una reducción de escala de los datos ERA mediante el modelo Weather Research and Forecasting o WRF (Skamarock et al., 2008), de hasta $4 \mathrm{~km}$ de resolución espacial, diferentes bases de datos satelitales, así como una base de datos globales y datos locales precipitación para la zona central de Chile $\left(34^{\circ} \mathrm{S}-41^{\circ} \mathrm{S}\right)$, desde enero del 2000 hasta diciembre del 2011, en la zona de estudio. Es decir, mucho más corta que nuestra base de datos.

Al comparar ambas series de precipitación se obtuvo un $\mathrm{R}^{2} \approx 0.88$ (Figura 4), que respalda el procedimiento de isoyetas efectuado anteriormente. 


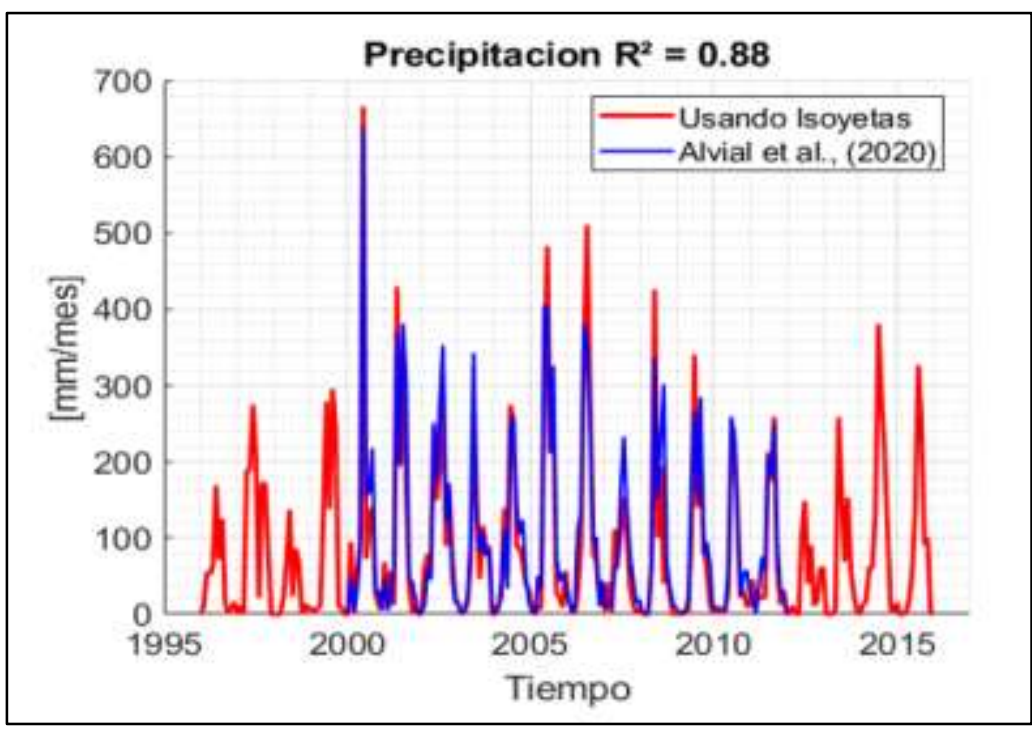

Figura 4. Comparación entre los Montos de Precipitación Obtenida por Medio de Isoyetas y la Serie desarrollada por Alvial et al. (2020) en Batuco.

Para la temperatura, cuya variabilidad espacial es más homogénea que la de la precipitación, no existiría necesidad de tantas estaciones de monitoreo (Garreaud et al., 2009). Esta sería la razón por la cual las estaciones de monitoreo de temperatura son escasas, particularmente en la región de estudio.

El criterio de selección para las estaciones de temperatura (la estación número 10 y desde la 20 hasta 23 del Cuadro 1) fue que se contara con disponibilidad de datos desde abril del año 2010 hasta abril del año 2021.

A diferencia de la precipitación, donde se calculó un valor anual representativo para cada estación (ecuaciones 10 y 11), en el caso de la temperatura se calculó un valor mensual representativo (análogo a la climatología) a partir de la ecuación (12):

$$
T C_{(p m, e)}=\frac{\sum_{a=1}^{N} T_{(m, a, e)}(a)}{n}
$$

Donde:

$\mathrm{TC}(\mathrm{pm}, \mathrm{e})=$ valor representativo de la temperatura promedio mensual para una estación e (en adelante y por simplicidad, la climatología de cada estación)

$\mathrm{T}(\mathrm{m}, \mathrm{a}, \mathrm{e})=$ valor de temperatura mensual de un mes $\mathrm{m}$, de un año a, de una estación e

$\mathrm{n} \quad=$ número de años.

Una vez calculada la climatología de temperatura en cada estación, con la ayuda de un sistema de información geográfica (SIG), se realizó un proceso similar al de la Figura 3, para obtener 12 mapas de isotermas representativas de cada mes.

En cada mapa de isotermas (12 en total) existe un valor representativo de la zona de estudio (valor climatológico) que corresponderá al mismo punto usado en la Figura $\mathrm{N}^{\circ} 3$.

Se escogió la estación Ninhue (estación número 22 del Cuadro 1) como la estación de referencia. Luego, a partir de la ecuación (13) se calculó la temperatura en Batuco a escala mensual. 


$$
T b_{(m, a)}=T n_{(m, a)} x \frac{T C b_{(m)}}{T C n_{(m)}}
$$

Donde:

$$
\begin{aligned}
& \mathrm{Tb}(\mathrm{m}, \mathrm{a})=\text { Temperatura en Batuco (zona de estudio) en el mes } m \text { y el año a } \\
& \mathrm{Tn}(\mathrm{m}, \mathrm{a})=\text { temperatura en el mes } \mathrm{m} \text { y el año a, de la estación de referencia (Ninhue) } \\
& \mathrm{TCb}_{(\mathrm{m})}=\text { temperatura climatológica representativo en el mes } \mathrm{m} \\
& \mathrm{TC}_{(\mathrm{pa})}=\text { temperatura climatológica de la estación de referencia (Ninhue) en el mes } \mathrm{m} .
\end{aligned}
$$

Una vez obtenidas las series de precipitación y temperatura in situ en la zona de estudio (precipitación de isoyetas en batuco y temperatura de isotermas en batuco de ahora en adelante), se procedió a usarlas como base para corregir el sesgo de las series de precipitación y temperatura Arclim.

Para ello se usó el método Quantile Mapping (e.g., Enayati et al., 2021) en donde a la serie de precipitación de isoyetas en batuco y la serie de precipitación Arclim se le ajustó a una distribución gamma mientras a la serie de temperatura de isotermas en batuco y a la serie de temperatura Arclim se le ajustó a una distribución de probabilidad gaussiana (Wilks, 2005).

En las figuras 5 y 6 se sintetiza el procedimiento efectuado para estimar precipitación y temperatura respectivamente. A la serie de Arclim (verde), se le aplica una corrección de sesgo en base a las series extraídas por el método de interpolación de isoyetas e isotermas, respectivamente (rojos).

Adicionalmente, se agregan las series extraídas in situ de las estaciones 24 y 25 de la microcuenca Batuco (negro) denominada INFOR, las que se usan como referencia visual para comparar con los datos considerados como reales en la zona de estudio.

Finalmente, las series de entrada al modelo Weap, tanto para la precipitación como para la temperatura, fueron las series Arclim corregidas de la Figuras 5 y 6 , respectivamente, señaladas en color azul.

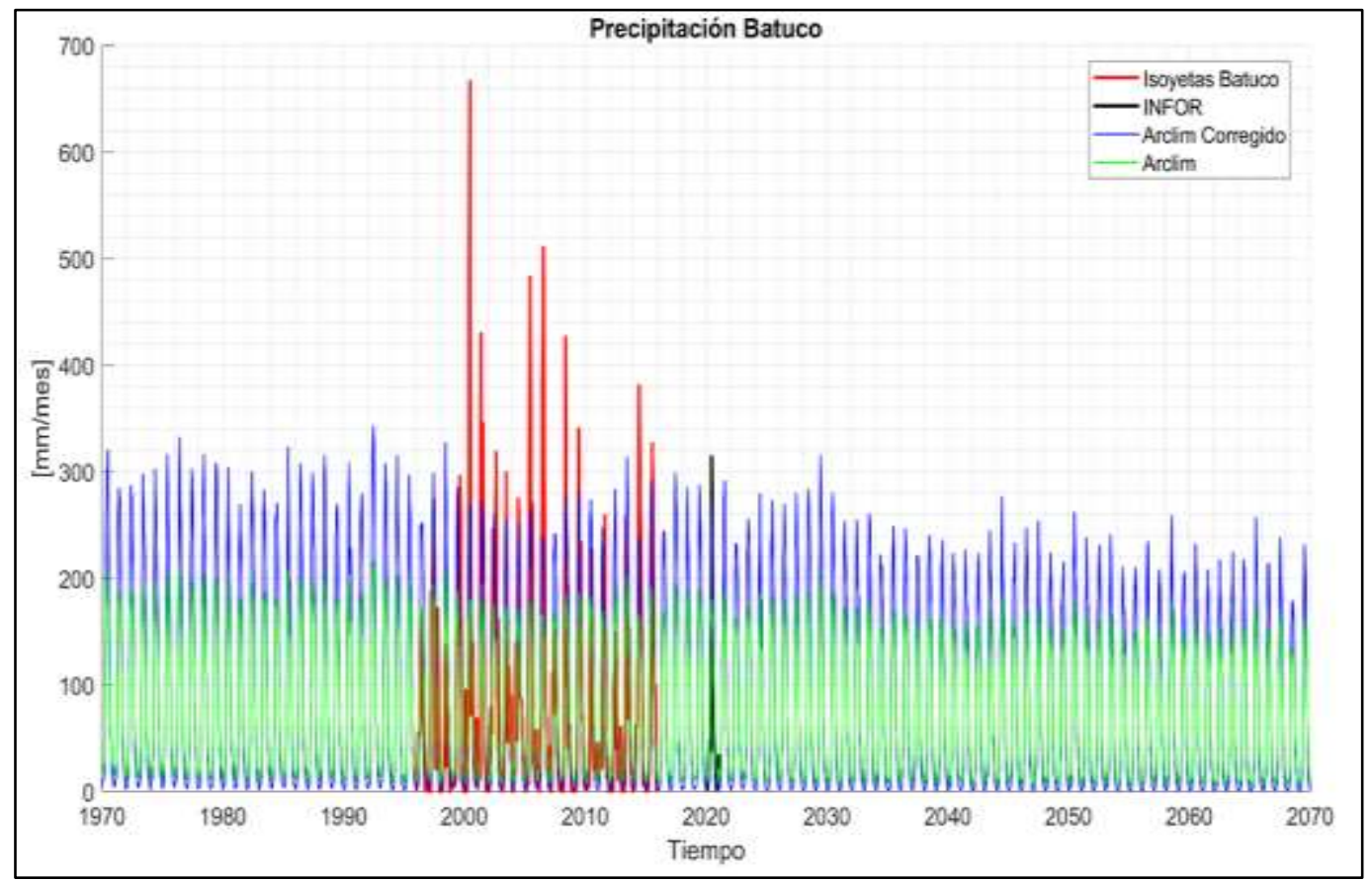

Figura 5. Distintas Series de Precipitación Disponibles para Batuco. 


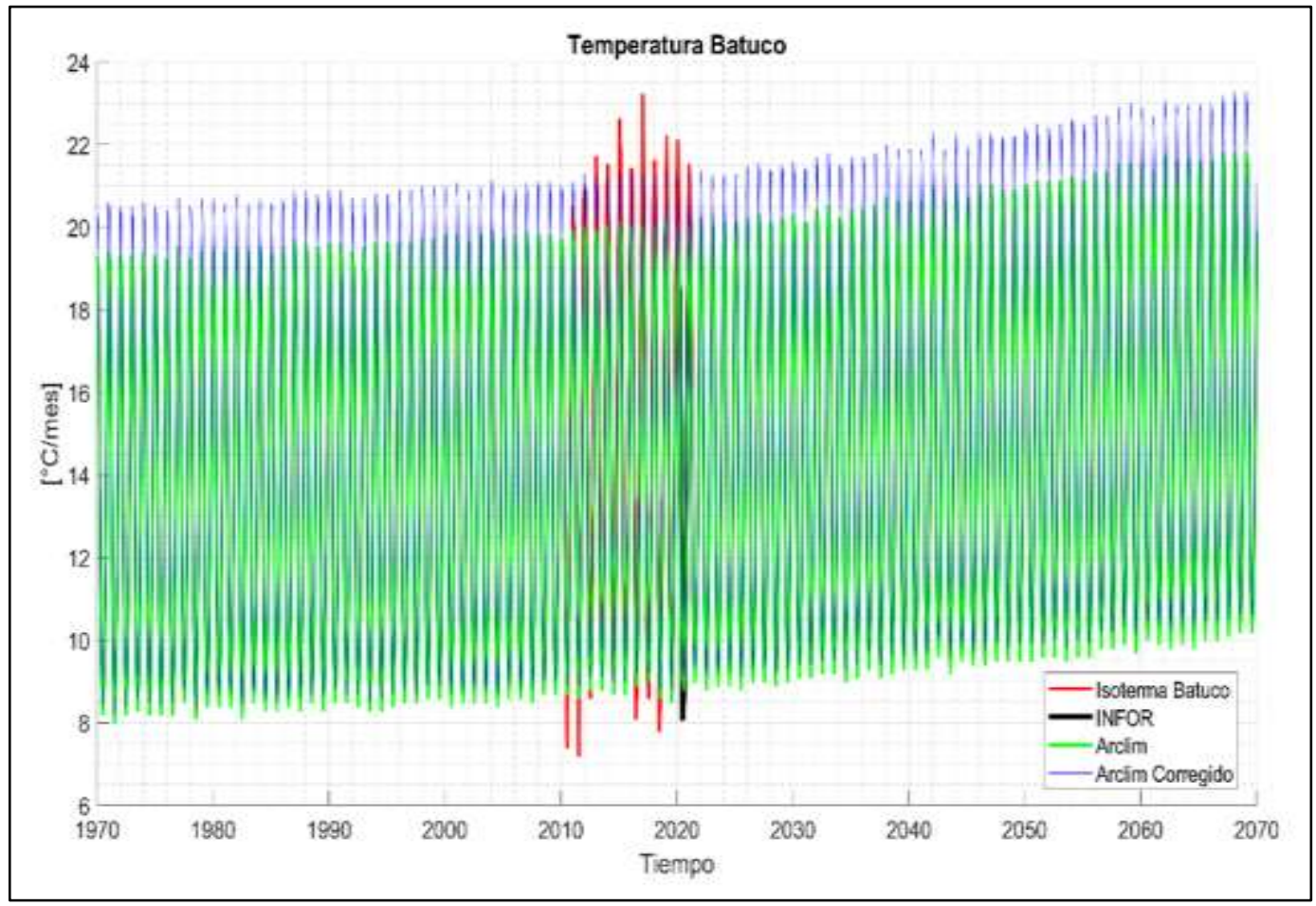

Figura 6. Distintas Series de Temperatura Disponibles para Batuco.

Se debe mencionar que tiene que existir cierto cuidado al trabajar con los datos Arclim. Center for Climate and Resilience Research (CR)2 et al. (2020) destacan con gran fuerza que incluso en el periodo histórico (1980-2009), los modelos no intentan ajustarse a las variaciones observadas, sino representar las condiciones promedio y su rango de variabilidad.

Por ejemplo, la temperatura máxima simulada sobre Santiago para el 27 de Julio de 1987 o la precipitación acumulada el año 2007 no guardan relación con lo observado. Sin embargo, la precipitación promedio anual entre 1980-2010 debe ser similar entre las observaciones y simulaciones.

\section{- Otras Variables Meteorológicas}

En el caso de las otras variables meteorológicas que son necesarias como entradas en la simulación hidrológica; la humedad relativa y la velocidad del viento fueron obtenidas desde estaciones de monitoreo de INFOR en Batuco (estaciones 24 y 25 del Cuadro 1), considerado la variabilidad anual del 2020 como un ciclo a repetir para todo el periodo de tiempo.

Respecto a la fracción de cobertura de nubes, se extrajo del producto climático de los datos de re análisis ERA5, calculando un promedio mensual del píxel que cubre la zona de estudio entre los años 2013 y 2015.

\section{Clases de Uso de Suelo}

Para no parametrizar en exceso el modelo, se separó la zona de estudio en 3 clases de usos de suelos:

-Una zona boscosa, con 164,57 ha.

-Una zona agrícola de praderas o pasturas naturales con 135,39 ha.

-Una zona urbana o sin vegetación con 236,90 ha (Figura 7). 


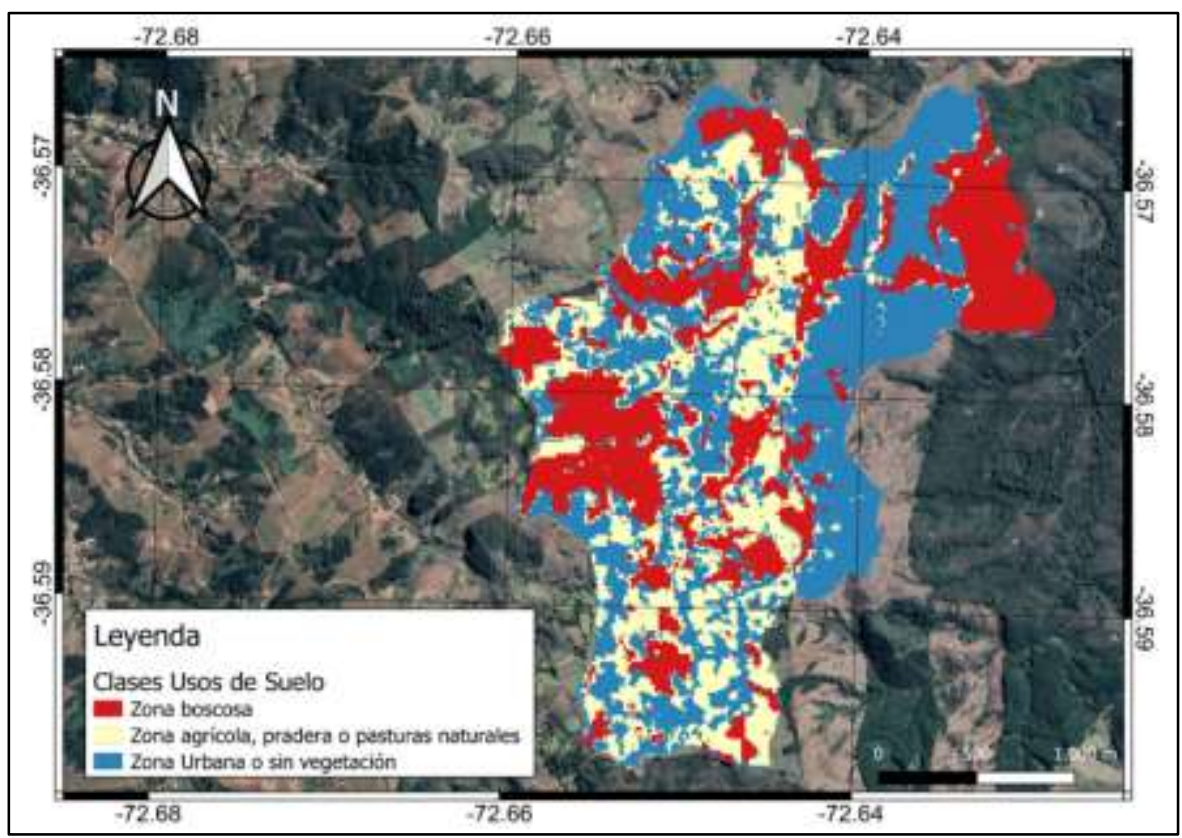

Figura 7. Clases de Uso del Suelo en Batuco

\section{Consideraciones Antes y Durante la Simulación Hidrológica}

Se considerará un periodo de prueba del modelo hidrológico 1970-1974, donde se buscó atenuar los efectos de las condiciones iniciales. Este periodo de tiempo no fue incluido dentro de los resultados, los cuales corresponden a enero de 1975 hasta diciembre del 2064.

Se usó los datos de la humedad del suelo (estación 26, Cuadro $N^{\circ} 1$ ) para hacer un tipo de calibración de la simulación hidrológica, con la humedad del tanque 1 y tanque 2 (Figura 2) simuladas. Se usó el coeficiente de determinación $\left(\mathrm{R}^{2}\right)$ para este propósito.

\section{RESULTADOS}

El caudal simulado marca una pendiente negativa en el periodo de estudio (enero de 1975 hasta diciembre de 2064$)$ de $\approx-6,43 \mathrm{~L} / \mathrm{s}$ por década. La varianza compartida $\left(R^{2}\right)$ entre la precipitación y el caudal simulado fue de $\approx 0,46$, mientras que la correlación entre la temperatura y el caudal simulado fue de $\approx-0,84$ (Figura 8, izquierda).

Para el promedio cada 10 años, el rango de valores fluctuó entre $\approx 82,5$ y $\approx 134,2 \mathrm{~L} / \mathrm{s}$.

Las AEProm10 (Anomalías estandarizadas del promedio cada 10 años) muestran un descenso sostenido a medida que avanzan las décadas, donde el periodo del 2060 está a $\approx-1,5$ desviaciones estándar con respecto a la media de la serie.

Por otra parte, AEPend10 (anomalías estandarizadas de las pendientes cada 10 años), presentan un comportamiento más cíclico, pero la amplitud va disminuyendo a medida que avanza el tiempo, donde en la década del 2060 está $a \approx 0$ desviaciones estándar con respecto a la media de la serie.

En los gráficos de la Figura 9 se desglosa el caudal simulado del estero batuco en sus correspondientes temporadas del año.

En el verano austral (DEF) el caudal simulado marca una pendiente de $\approx-2,29 \mathrm{~L} / \mathrm{s}$ por década, el $\mathrm{R}^{2}$ entre la precipitación y el caudal simulado de verano fue de $\approx 0,58$, mientras que la correlación entre la temperatura y el caudal simulado de verano fue de $\approx-0,95$.

El promedio por cada 10 años tomó el rango desde $\approx 38,7 \mathrm{~L} / \mathrm{s}$ hasta $\approx 18,2 \mathrm{~L} / \mathrm{s}$. 


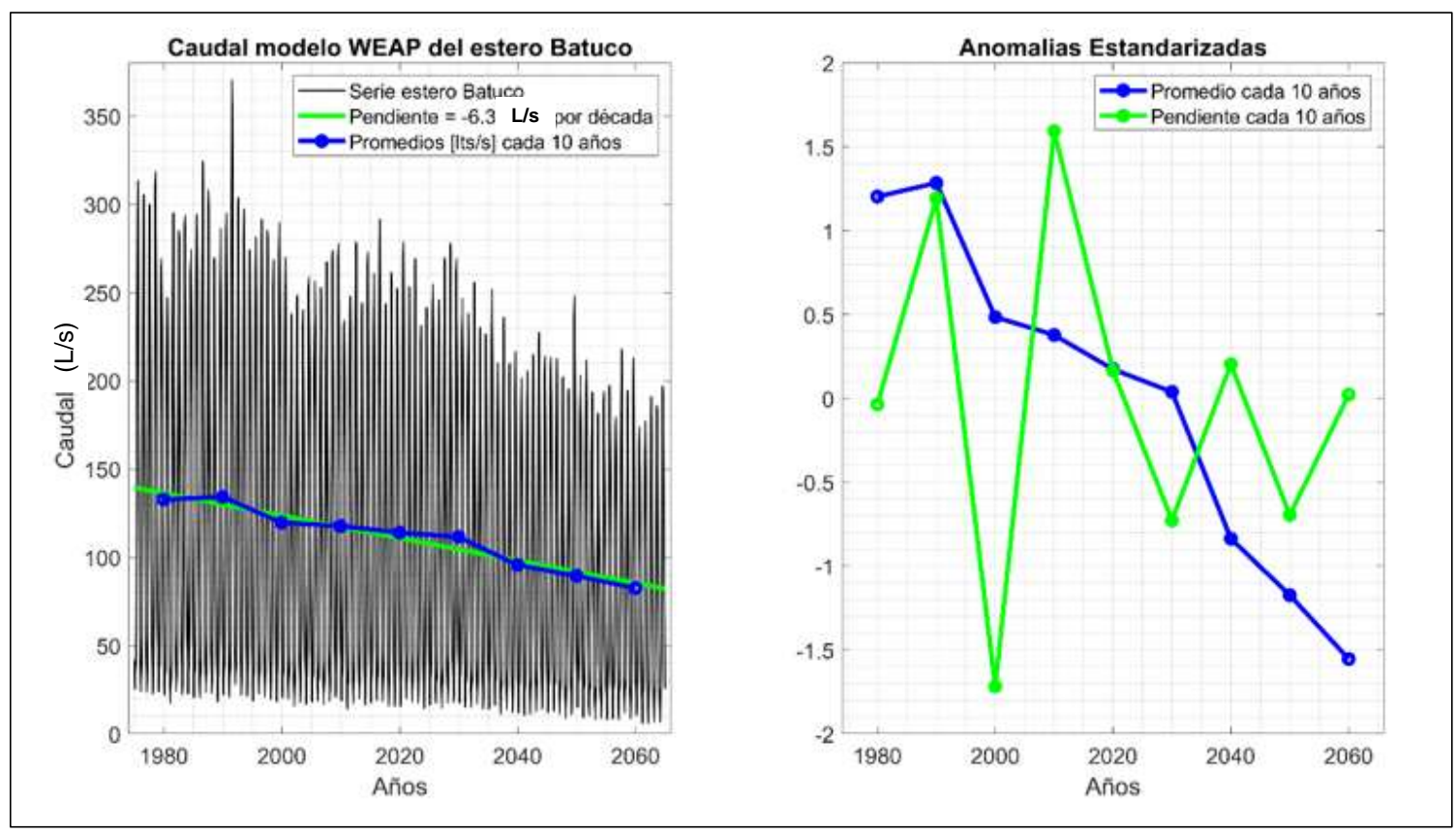

Figura 8. Series de Caudal Simulado del Estero Batuco (negro), pendiente (verde) y promedios cada 10 Años (azul) (Izq.). Anomalías Estandarizadas de los Promedios cada 10 Años (azul) y Pendientes cada 10 Años (verde) (Der.)

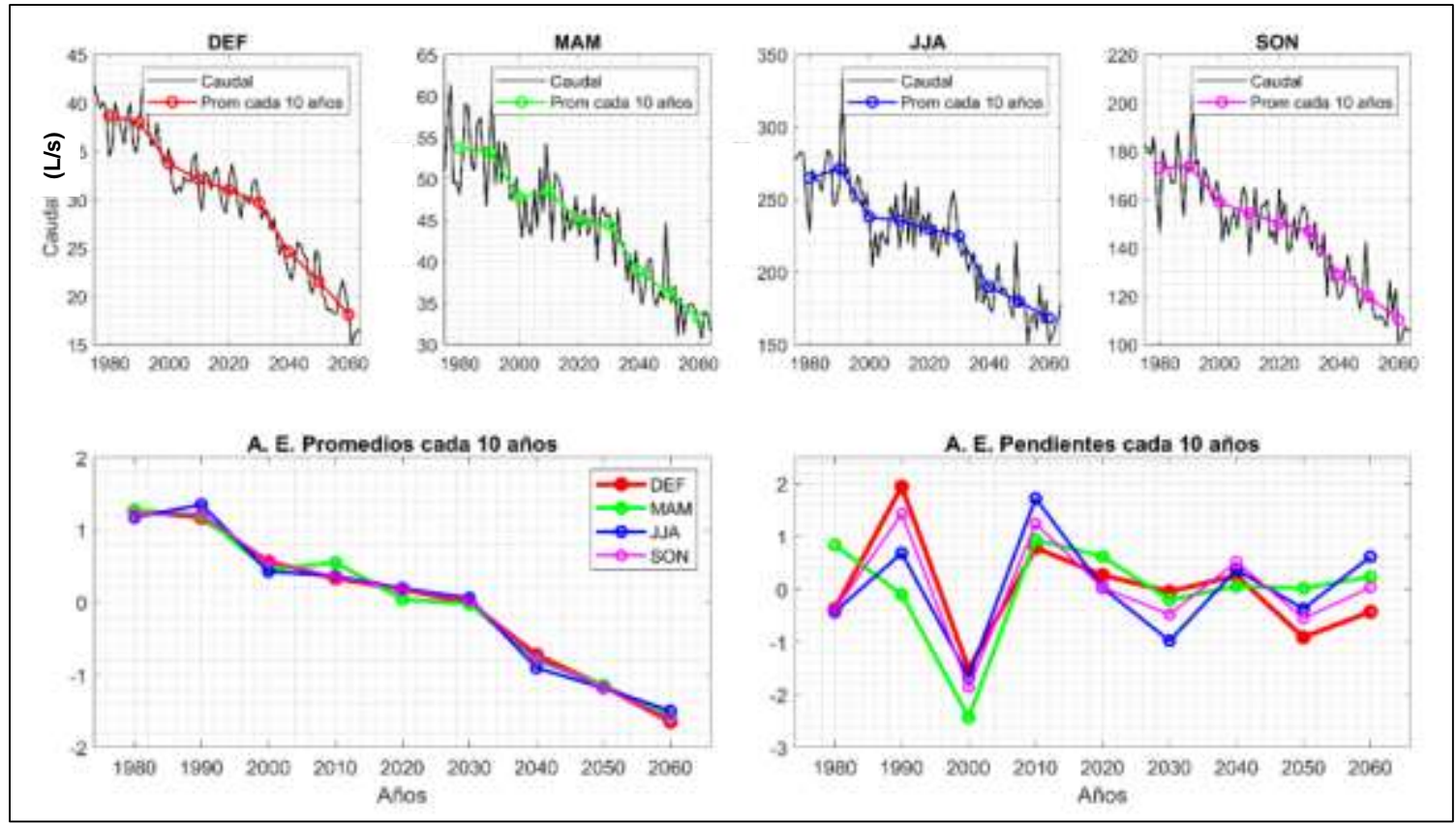

Figura 9. Caudal Estacional y Promedios cada 10 Años (arriba) A.E Anomalías Estandarizadas. A.E. de los Promedios cada 10 Años (abajo izq.) y Pendientes cada 10 Años (abajo der.)

En el otoño austral (MAM) el caudal simulado marca una pendiente de $\approx-2,32 \mathrm{~L} / \mathrm{s}$ por década, el $\mathrm{R}^{2}$ entre la precipitación y el caudal simulado de otoño fue de $\approx 0,93$, mientras que la correlación entre la temperatura y el caudal simulado de otoño fue de $\approx-0,88$. El promedio por cada 10 años tiene un rango desde $\approx 53,8 \mathrm{~L} / \mathrm{s}$ hasta $\approx 33,2 \mathrm{~L} / \mathrm{s}$. 
En el invierno austral (JJA) el caudal simulado marca una pendiente de $\approx-11,6 \mathrm{~L} / \mathrm{s}$ por década, el $\mathrm{R}^{2}$ entre la precipitación y el caudal simulado de invierno fue de $\approx 0,92$, mientras que la correlación entre la temperatura y el caudal simulado de invierno fue de $\approx-0,86$. El promedio por cada 10 años tiene un rango desde $\approx 265,1 \mathrm{~L} / \mathrm{s}$ hasta $\approx 168,4 \mathrm{~L} / \mathrm{s}$.

En la primavera austral (SON) el caudal simulado marca una pendiente de $\approx-7,25 \mathrm{~L} / \mathrm{s}$ por década, el $\mathrm{R}^{2}$ entre la precipitación y el caudal simulado de primavera fue de $\approx 0,77$, mientras que la correlación entre la temperatura y el caudal simulado de primavera fue de $\approx-0,91$. El promedio por cada 10 años tomó el rango desde $\approx 173,9 \mathrm{~L} / \mathrm{s}$ hasta $\approx 110,4 \mathrm{~L} / \mathrm{s}$.

Las anomalías estandarizadas promedios cada 10 años, para las diferentes temporadas (DEF, MAM, JJA y SON) se presentan en el gráfico inferior izquierdo de la Figura 9. En él se observa que, en la medida que avanzan las décadas, todas las temporadas exhiben un descenso sostenido del caudal, respecto a la media de cada serie. En la década del 2060 está entre -1,4 y -1,6 desviaciones estándar con respecto a las medias de cada serie, siendo la temporada de invierno (JJA) la más cercana a su media y la de verano (DEF) la que exhibe el mayor descenso respecto a la media de la serie.

En el gráfico inferior derecho de la Figura 9, se pueden ver las AEPend10 de las diferentes temporadas del año. Se observó un comportamiento cíclico cuya amplitud va disminuyendo a medida que avanzan las décadas. En el periodo del 2060 las temporadas invernales (JJA) están a $\approx 0,6$ desviaciones estándar con respecto a la media de su serie, mientras que en las temporadas de verano (DEF) están $a \approx-0,4$ desviaciones estándar con respecto a la media de serie.

\section{DISCUSION Y CONCLUSIONES}

La simulación efectuada constituye una primera aproximación a las tendencias que manifiesta la microcuenca Batuco y podrá ser calibrada y validada en la medida que se disponga de datos de caudal observado (SEI, 2015), que en la actualidad no existen. Como medida paliativa se efectuó un ajuste con datos de humedad del suelo in situ (estación 26 del Cuadro 1), donde se obtuvo para el tanque 1 (representación Figura 2), para la zona boscosa un $\mathrm{R}^{2}$ de $\approx 0,57$; mientras que para el tanque 2 , el $\mathrm{R}^{2}$ fue $\mathrm{de} \approx 0,88$. Este procedimiento no reemplaza a la calibración con valores de caudal observado por cuanto no considera la variabilidad interanual e interdecenios, el rango de amplitudes del caudal simulado, y tampoco lo que ocurre en las otras zonas de uso de suelo (SEI, 2015), pero aun así es un elemento que contribuye a mejorar la estimación obtenida en la simulación.

La simulación del caudal del estero Batuco muestra una tasa de cambio o pendiente negativa de $\approx-6,3$ $\mathrm{L} / \mathrm{s}$ por década, lo cual es consistente con el escenario del cambio climático RCP 8.5 , donde se estima que en el centro de Chile existiría un descenso en la cantidad de precipitaciones, un aumento en las temperaturas y, por consiguiente, una reducción general del caudal de los ríos y cursos de agua (e.g., Mena, 2009; Río La Mura, 2015; Vargas et al., 2012).

Debido a que la varianza compartida del caudal simulado con la precipitación es de $\approx 50 \%$, el caudal del estero Batuco sería medianamente sensible a las variaciones y cambios que la precipitación experimente en el futuro.

Considerando las limitaciones del estudio no se debe considerar la cuantía exacta de los valores obtenidos como resultados de promedios ni pendientes. Ellos deben tomarse como referencias indicadoras de tendencias generales en determinados intervalos de tiempo, pero no como valores absolutos. Con esta finalidad se calcularon las AEProm10 y las AEPend10, para observar su comportamiento o tendencia con respecto a sus medias, a medida que avanza el tiempo y no para interpretar sus valores específicos.

En el caso de las AEProm10 de la serie completa (Figura 8), independiente de sus valores específicos, se observa un descenso sostenido con respecto a la media de la serie, indicando que los caudales han disminuido y seguirán disminuyendo, independientemente de que se conozcan o no sus valores reales.

En el caso de las AEPend10 (Figura 8) se observa un comportamiento cíclico donde la máxima distancia positiva con respecto a su media fue en el periodo del 2010, mientras que la máxima distancia negativa fue en la década del 2000. Este comportamiento cíclico se mantiene a través del tiempo, pero su amplitud va disminuyendo. 
Las anomalías estandarizadas del caudal simulado en cada época del año (Figura 9) no varían entre ellas, tienen el mismo comportamiento en el tiempo y además coinciden con la tendencia de la serie completa que se muestra en la Figura 8.

En el caso de las AEProm10 no existe una diferencia mayor 0,3 desviaciones estándar entre las distintas temporadas del año y el comportamiento es similar a medida que avanzan los años.

El descenso de caudal en el tiempo se puede deducir a partir del Cuadro 3, donde se muestran Prom10 (promedios cada 10 años) y Pend10 (tendencias cada 10 años), tanto para la serie completa como para cada temporada del año. Estos valores deben tomarse con cuidado y solo de manera referencial, pues como se indicó anteriormente su finalidad es solo evidenciar la similitud en el comportamiento de la variabilidad de cada serie y no el valor absoluto de las mismas.

Cuadro 3. Promedios y Desviaciones Estándar de los Promedios y Pendientes cada 10 Años.

\begin{tabular}{lrrrr}
\hline \multirow{2}{*}{ Temporada } & \multicolumn{2}{c}{ Media (1975-2064) } & \multicolumn{2}{c}{ Desviación estándar (1975-2064) } \\
\cline { 2 - 5 } & Prom10 [L/s] & Pend10 [L/s] & Prom10 [L/s] & Pend10 [L/s] \\
\hline Todo el año & 110,79 & 3,86 & 18,19 & 8,42 \\
\hline DEF & 29,74 & $-3,05$ & 7,05 & 2,28 \\
\hline MAM & 44,55 & $-1,79$ & 7,21 & 3,33 \\
\hline JJA & 222,54 & $-16,55$ & 36,23 & 18,88 \\
\hline SON & 146,34 & $-11,05$ & 22,38 & 7,60 \\
\hline
\end{tabular}

Los resultados presentados corresponden a una primera aproximación a la simulación hidrológica de la microcuenca Batuco. Para futuros estudios en esta materia es recomendable que se generen datos de caudal, los que podrían obtenerse con la instalación de un correntómetro u otro sensor, o alternativamente usando un método indirecto, como la aplicación de Android denominada DiScHaRgE (Carrel et al., 2019). Disponer de estos datos para periodos de al menos un año, de modo que capturen la variabilidad intra-anual, permitiría calibrar la simulación hidrológica (e.g., Nash and Sutcliffe, 1970), sin embargo, tal información actualmente es inexistente.

Otra opción para mejorar la precisión de futuras simulaciones hidrológicas en la zona de estudio, es esperar a que las estaciones meteorológicas de INFOR acumulen registros in situ por más de 3 años, situación que se cumplirá entre los años 2023 y 2024. Esta información permitirá estimar no solo la variabilidad intra-anual, sino que también la variabilidad interanual, sin la necesidad de recurrir a otras bases de datos de reanálisis como entrada principal a una microcuenca más pequeña que un pixel en algunos productos climáticos.

Independiente de lo anterior, los datos presentados permiten identificar una tendencia en el comportamiento hidrológico de la microcuenca Batuco y su evolución durante el siglo XXI, constituyendo un antecedente para analizar o planificar futuras medidas hidrosociales para beneficio de los habitantes de la zona. Asociado al sostenido descenso que se proyecta para el caudal y considerando que la zona de Batuco depende del comportamiento hídrico in situ (Vargas et al., 2019), se espera que la menor disponibilidad de agua generará un creciente estrés hídrico en la población. Actualmente (2021) se encuentran en ejecución obras de un proyecto de agua potable rural (SIMOL et al., 2021), no obstante, resulta recomendable considerar también la construcción de colectores de lluvia y canales de regadío, con el fin de reforzar no solo el consumo poblacional, sino también reforzar los sectores productivos agrícola y ganadero.

\section{AGRADECIMIENTOS}

Los autores agradecen al proyecto ANID/FONDEF ID19I10121 "Sistema de monitoreo para la participación local en la gestión integrada de cuencas" (SIMOL), y al programa Minagri: "Monitoreo de cuencas y otros ecosistemas forestales vinculados al consumo humano del agua en la zona centro sur de Chile" (Código 3041331211), por permitir y apoyar la elaboración de esta investigación. 


\section{REFERENCIAS}

Allen, R., Pereira, L., Rae, D. \& Smith, M. (1998). Crop Evapotranspiration Guidelines for computing crop water requeriments-FAO Irrigation and Drainage Paper 56. FAO, Rome, 300(9): D05109.

Alvial, F., Abarca-del-Río, R. \& Ávila, A. (2020). High-Resolution Precipitation Gridded Dataset on the South-Central Zone (34 S-41 S) of Chile. Frontiers in Earth Science. https://doi:10.3389/feart.2020.519975.

Bhave, A., Mishra, A. \& Raghuwanshi, N. (2014). A combined bottom-up and top down approach for assessment of climate change adaptation options. J. Hydrol., 518. Pp: 150-161. https://doi:10.1016/j.jhydrol.2013.08.039.

Boisier, J., Rondanelli, R., Garreaud, R. \& Muñoz, F. (2016). Anthropogenic and natural contributions to the Southeast Pacific precipitation decline and recent mega drought in central Chile. Geophysical Research Letters, 43(1): 413-421. https://doi.org/10.1002/2015gl067265.

Bozkurt, D., Rojas, M., Boisier, J. \& Valdivieso, J. (2017). Climate change impacts on hydroclimatic regimes and extremes over Andean basins in central Chile. Hydrology and Earth System Sciences Discussions. https://doi.org/10.5194/hess-2016-690.

Carrel, M., Detert, M., Peña-Haro, S. \& Luethi, B. (2019). Evaluation of the DischargeApp: A Smarthphone Application for Discharge Measurements. HydroSentSoft 2019, Madrid, Spain. En: https://discharge.ch/pdf/Carrel et al 2019.pdf.

Center for Climate and Resilience Research (CR)2, Centro UC Cambio Global (CCG), Ministerio del Medio Ambiente (MMA) \& Ministerio Federal de Cooperación Económica y Desarrollo (BMZ) de Alemania. (1970-2069). ARCLIM Explorador de Amenazas Climáticas. Precipitación y Temperatura Mensual. En: https://arclim.mma.gob.cl/amenazas/

Center for Climate and Resilience Research (CR)2, Centro UC Cambio Global (CCG), Ministerio del Medio Ambiente (MMA) \& Ministerio Federal de Cooperación Económica y Desarrollo (BMZ) de Alemania. (2020). Explorador de Amenazas Climáticas. Descripción de la base de datos. Atlas de Riesgos Climáticos. En: https://arclim.mma.gob.cl/media/learning/Datos Climaticos.pdf.

Center for Climate and Resilience Research (CR)2, Dirección General de Aguas (DGA), Ministerio del Medio Ambiente (MAM), National Oceanic and Atmospheric Administration (NOAA) \& Dirección Meteorológica de Chile (DMC). (1940-2021). Explorador Climático [Precipitación Mensual]. En: https://explorador.cr2.cl/.

Centro de Cambio Global-Universidad Católica de Chile u Stockholm Environment Institute. (2009). Guía Metodológica - Modelación Hidrológica y de Recursos Hídricos con el Modelo WEAP. Desarrollada con contribuciones del PACC (Proyecto de Adaptación al Cambio Climático a través de una efectiva gobernabilidad del agua en Ecuador), Ministerio del Ambiente de Ecuador, y PROMAS (Programa para el Manejo del Agua y del Suelo) de la Universidad de Cuenca, Ecuador. En: https://cambioglobal.uc.cl/images/publicacionesextension/9 2009 Guia-para-la-modelacion-con-laplataforma-WEAP CCG-SEI.pdf.

Condom, T., Escobar, M., Purkey, D., Pouget, J., Suarez, W., Ramos, C. et al. (2011). Modelling the hydrologic role of glaciers within a water evaluation and planning system (WEAP): A case study in the Rio Santa watershed (Peru). Hydrol. Earth Syst. Sci. Discuss., 8. Pp: 869-916. https://doi.org/10.5194/hessd-8-869-2011.

Copernicus, European Commission \& European Centre for Medium-Range Weather Forecasts (ECMWF). (19792021). Copernicus Climate Data Store | Copernicus Climate Data Store [Total cloud cover]. En: https://cds.climate.copernicus.eu/cdsapp\#!/dataset/reanalysis-era5-single-levels-monthlymeans?tab=overview

Copernicus \& European Space Agency (ESA). (2015). Copernicus Open Access Hub. Imágenes Satelitales. En: https://scihub.copernicus.eu/dhus/\#/home.

Davie, T. (2008). Streamflow Analysis and Modelling: Fundamentals of Hydrology. Second Edition. Pp: 101-124.

Duque, L. \& Vázquez, R. (2015). Modelación de la oferta hídrica en una cuenca de montaña tropical en función de su cobertura del suelo. Water Availability Modelling for a Tropical Mountain Catchment as a Function of its Soil Cover. Aqua-LAC, 7(1): 63-76. https://doi.org/10.29104/phi-aqualac/2015-v7-1-08 
Enayati, M., Bozorg-Haddad, O., Bazrafshan, J., Hejabi, S. \& Chu, X. (2021). Bias correction capabilities of quantile mapping methods for rainfall and temperature variables. Journal of Water and Climate Change, 12(2): 401419. https://doi.org/10.2166/wcc.2020.261

Fernández-Alberti, S., Abarca-del-Río, R., Bornhardt, C. \& Ávila A., 2021. Development and Validation of a Model to Evaluate the Water Resources of a Natural Protected Area as a Provider of Ecosystem Services in a Mountain Basin in Southern Chile. Front. Earth Sci., 8:539905. https://doi.org/10.3389/feart.2020.539905

Garreaud, R.D., Vuille, M., Compagnucci, R. \& Marengo, J. (2009). Present-day South American climate. Palaeogeography, $\quad$ Palaeoclimatology, $\quad$ Palaeoecology, $\quad 281(3-4): \quad$ 180-195. https://doi.org/10.1016/i.palaeo.2007.10.032

Garreaud, R.D. (2014). La mega sequía 2011-2013(?): Aspectos climáticos regionales de Chile.

Geophysical Institute of the University of Alaska Fairbank. (2006-2011). ASF Data Search [DEM (12.5m x 12.5m) Alos Palsar]. Disponible en: https://search.asf.alaska.edu/\#/?dataset=ALOS\&zoom=6.256\%C2\%A2er\%3D72.178,\%20-36.280.

Instituto de Investigaciones Agropecuarias (INIA), Centro de Estudios Avanzados en Zonas Áridas (CEAZA) \& Dirección Meteorológica de Chile (DMC). (2009-2021). Agrometeorología. INIA (Temperatura Mensual). En: https://agrometeorologia.cl/.

IPCC. (2014). Cambio climático 2014: Informe de síntesis. Contribución de los Grupos de trabajo I, II y III al Quinto Informe de Evaluación del Grupo Intergubernamental de Expertos sobre el Cambio Climático [Equipo principal de redacción, R.K. Pachauri y L.A. Meyer (eds.)]. IPCC. Ginebra, Suiza. 157 p. En: https://www.ipcc.ch/site/assets/uploads/2018/02/SYR AR5 FINAL full es.pdf.

IPCC. (2019). Socio-Economic Data and Scenarios. The Intergovernmental Panel on Climate Change. En: https://sedac.ciesin.columbia.edu/ddc/ar5 scenario process/RCPs.html.

Maliehe, M. \& Mulungu, D. (2017). Assessment of water availability for competing uses using SWAT and WEAP in South Phuthiatsana catchment, Lesotho. Phys. Chem. Earth, 100. Pp: 305-316. https://doi.org/10.1016/i.pce.2017.02.014

Marticorena, J. (2020). Consumo de Agua Promedio por Persona en Chile Supera la Media de Europa, aunque está muy por debajo de la de Estados Unidos. El Mercurio. En: https://www.litoralpress.cl/paginaconsultas/Servicios NClip/Get Imagen Pagina.aspx?LPKey=LVISR6H4DB 52E74KGLFSSNDHZAN3KR7H7FG7HH54BLBGYWWMBWKQ

Mena Pardo, D. (2009). Análisis de Impactos del Cambio Climático en la Cuenca Andina del Río Teno, Usando el Modelo Weap. En: https://repositorio.uchile.cl/handle/2250/103570.

Meza, F., Vicuña, S., Jelinek, M., Bustos, E. \& Bonelli, S. (2014). Assessing water demands and coverage sensitivity to climate change in the urban and rural sectors in central Chile. Journal of Water and Climate Change, 5(2): 192-203. https://doi.org/10.2166/wcc.2014.019

Ministerio de Agricultura, Universidad de Birmingham \& Imperial College de Londres. (2016). Análisis de vulnerabilidad ante la sequía agrícola en Chile. Proyecto "Apoyo al manejo efectivo de la gestión del riesgo de sequías en cuencas de Chile". En: http://www.climatedatalibrary.cl/UNEA/maproom/Vulnerability/Agricultural/Informe final Indice Vulnerabilida d julio2016.pdf.

Municipalidad de Ránquil. (2017). Actualización plan de desarrollo comunal de Ránquil 2016-2021.

Municipalidad de Ránquil. (2021). Actualización cantidad de habitantes en la microcuenca Batuco.

Murphy, J.M. (1988). The impact of ensemble forecasts on predictability. Quarterly Journal of the Royal Meteorological Society, 114(480): 463-493. https://doi.org/10.1002/qj.49711448010

Nash, J.E. \& Sutcliffe, J.V. (1970). River flow forecasting through conceptual models' part I-A discussion of principles. Journal of hydrology, 10(3): 282-290. https://doi.org/10.1016/0022-1694(70)90255-6

Río La Mura, P. (2015). Análisis del impacto del cambio climático en la disponibilidad del recurso hídrico en la Cuenca de Pangal en Pangal. En: https://repositorio.uchile.cl/handle/2250/135204. 
SEI. (2015). WEAP Water Evaluation and Planning System User Guide. Stockholm Environment Institute, 187 p. En: https://www.weap21.org/downloads/WEAP User Guide.pdf.

SIMOL (2021). Agencia Nacional de Investigación y Desarrollo, Gobierno de Chile, Universidad de Concepción, y Comités de agua potable rural (APR). Situación del Acceso al agua en comités locales de la comuna de Ránquil. Proyecto FONDEF ID19I10121. En: https://sistemademonitoreolocal.udec.cl/wpcontent/uploads/2021/05/INFORME-DIAGNOSTICO-COMITES.pdf.

Skamarock, W.C., Klemp, J.B., Dudhia, J., Gill, D.O., Barker, D.M., Duda, M.G. et al. (2008). A description of the advanced research WRF version 3. University Corporation for Atmospheric Research. https://dx.doi.org/10.5065/D68S4MVH.

Trenberth, K.E. (2011). Changes in precipitation with climate change. Climate Research, 47(1-2): 123-138. https://doi.org/10.3354/cr00953.

Vargas, V., Carrasco, N. \& Vargas, C. (2019). Local Participation in Forest Watershed Management: Design and Analysis of Experiences in Water Supply Micro-Basins with Forest Plantations in South Central Chile. Forests. 2019, 10, 580. https://doi.org/10.3390/f10070580.

Vargas, X., Ayala, A., Meza, R. \& Rubio, E. (2012). Disponibilidad futura de los recursos hídricos frente a escenarios de cambio climático en Chile. Disponible en: https://repositorio.cepal.org//handle/11362/5691.

Wilks, D.S. (2005). Parametric Probability Distribution: Statistical methods in the atmospheric sciences. Pp: 71-130. 